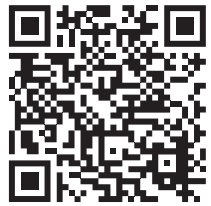

* Departamento de

Hemodinámica, Instituto Nacional de Cardiología Ignacio Chávez (INCOR). Ciudad de México, México.

‡ Servicio de Hemodinámica del INCOR. Hospital CIMA Hermosillo, Sonora.

$\S$ Cardiólogo

Intervencionista, adscrito a la LSCV

Centro Médico

ABC. Ciudad de

México, México.

॥ Cardiología

Intervencionista,

Departamento

de Cardiología y

Profesorado de

la Residencia de

Cardiología Clínica,

Hospital Central «Dr.

Ignacio Morones

Prieto», Facultad

de Medicina de la

Universidad Autónoma

de San Luis Potosí, SLP.

"Servicio de

Hemodinámica

y Cardiología

Intervencionista,

Instituto de Corazón

de Querétaro.

Querétaro, México.

** Subdirección de

Médicos Residentes de Hemodinámica,

Instituto Nacional de

Cardiología «Ignacio

Chávez». Ciudad de

México, México.

Recibido:

20/04/2020

Aceptado:

27/04/2020

\title{
Protocolos de atención de pacientes y medidas de seguridad personal de los profesionales de la salud en salas de cateterismo cardiaco durante el brote de COVID-19
}

\author{
Patient care protocols and personal safety measures of health professionals \\ in cardiac catheterization departments during the COVID-19 outbreak
}

Guering Eid-Lidt,* Jorge Cortés-Lawrenz, ${ }^{\ddagger}$ Julio López-Cuellar, ${ }^{\S}$ José L Leiva-Pons,॥ Marco A Alcocer-Gamba,` Julio I Farjat-Pasos,** Juan A García-Alcántara ${ }^{\ddagger}$

\section{INTRODUCCIÓN}

Panorama

$\mathrm{L}$ a enfermedad por coronavirus 2019 (COVID-19), causada por el nuevo coronavirus 2 del síndrome respiratorio agudo grave (SARSCoV-2) fue descrita por primera vez a finales de $2019{ }^{1}$ se transformó rápidamente en una emergencia de salud pública y fue declarada pandemia por la Organización Mundial de la Salud (OMS) el 11 de marzo de $2020 .^{2}$

El primer caso en nuestro país fue diagnosticado el 28 de febrero de $2020 ;^{3} \sin$ embargo, para el día 16 de abril se estimaban ya 55,951 casos. ${ }^{4}$ Estas cifras traducen la alta transmisibilidad que este virus tiene entre los seres humanos, lo que representa una alta carga de morbimortalidad, así como un alto riesgo de contagio comunitario. Sin embargo, existe mayor riesgo de contagio entre los profesionales del área de la salud durante la atención de estos pacientes, sea en un triaje respiratorio, una terapia intensiva o una sala de cateterismo cardiaco.

\section{Dilema de riesgos en la} intervención cardiológica

El alto grado de contagio de esta enfermedad obliga a adoptar medidas de seguridad para proteger la salud de todos los pacientes y los profesionales del área de la salud que están expuestos a ella. Las salas de cateterismo cardiaco no son la excepción. Esto genera un nuevo dilema en la atención de los pacientes que serán llevados a un procedimiento invasivo cardiovascular. Por un lado, se debe minimizar la exposición de pacientes de riesgo a la enfermedad COVID-19, prevenir contagios del personal de salud, ${ }^{5}$ y emplear de manera racional los recursos disponibles, ${ }^{6}$ y todo esto manteniendo siempre una alta calidad en la intervención cardiológica. Por otro lado, se tienen que seguir realizando los procedimientos de intervención cardiovascular que sean necesarios para incidir en la morbimortalidad de los pacientes con afecciones cardiovasculares agudas y crónicas que ameritan una intervención, incluso en tiempos de contingencia por este nuevo brote. ${ }^{7}$

Objetivo: Este documento se creó para informar y capacitar al personal que labora en las salas de cateterismo cardiaco sobre los riesgos así como el plan de contención, mitigación y respuesta a la situación global y local de la infección por SARS-CoV-2. La orientación proporcionada en este documento se basa en la evidencia mundial más reciente de fuentes médicas y científicas que rigen la salud global, tales como la OMS y las principales asociaciones de profesionales cardiovasculares del mundo, quienes actual- 
mente se enfrentan ya pasada la epidemia y otras en fases avanzadas del brote de esta enfermedad, y que cuentan con la experiencia y la evidencia necesaria para emitir estas recomendaciones, mismas que han sido adaptadas a nuestro contexto y pueden ser adoptadas de manera universal.

\section{PROTOCOLOS DE ATENCIÓN DE PACIENTES}

\section{Recomendaciones internacionales}

Múltiples sociedades nacionales e internacionales han adaptado sus protocolos de atención y de seguridad de acuerdo con lo que recomienda la OMS. Destacan recomendaciones de la Sociedad China de Cardiología, ${ }^{8}$ el Sistema Nacional de Salud de Londres así como consensos del American College of Cardiology y la Society for Cardiovascular Angiography and Interventions ${ }^{7}$ (incluyendo recomendaciones en procedimientos estructurales), ${ }^{9}$ la Asociación del Ritmo Cardiaco, la Asociación de Cardiología Intervencionista y la Sociedad Española de Cardiología. ${ }^{10}$

Conjuntando las recomendaciones de la OMS ${ }^{5,6,11-15}$ y de todas éstas y otras asociaciones, ${ }^{16,17}$ describimos a continuación los protocolos de atención de los tres grandes grupos de pacientes que se atienden en las salas de cateterismo cardiaco, siempre tomando en cuenta que se deben seguir las medidas de seguridad y de protección personal señaladas más adelante, así como tomar en cuenta que todo paciente se debe considerar portador asintomático hasta no demostrar lo contrario.

\section{Síndromes isquémicos coronarios agudos}

Debe priorizarse la intervención coronaria percutánea primaria (ICPp) del infarto agudo de miocardio con elevación del segmento ST (IAMCEST), 7,8,16 sobre todo aquellos de localización anterior y extenso de menos de 12 horas de evolución o aquellos que tengan contraindicación mayor para trombólisis. Tanto para pacientes con IAMCEST, infarto agudo de miocardio sin elevación del segmento ST (IAMSEST) o angina inestable de alto riesgo se deberá priorizar la intervención coronaria percutánea urgente en los casos que presenten inestabilidad hemodinámica o eléctrica, y/o angina persistente. En pacientes con trombólisis fallida se debe preferir la intervención coronaria percutánea de rescate (ICPr). Aquellos pacientes con IAMSEST y neumonía grave, miocarditis sin inestabilidad hemodinámica, infarto de miocardio tipo 2 por sepsis, respuesta inflamatoria sistémica o lesión miocárdica o IAMSEST de bajo riesgo se recomienda evaluar caso por caso y estimar la posibilidad de diferir la intervención coronaria percutánea según sea el caso (Figura 1).

\section{Cardiopatías estructurales}

La mayoría de los centros de procedimientos estructurales en el mundo han cerrado sus programas de intervención estructural; sin embargo, existen ciertas condiciones en las que se deberá priorizar llevar al paciente a este tipo de procedimientos: ${ }^{9}$ pacientes con estenosis aórtica (EA) grave en clase funcional III/IV de la New York Heart Association (NYHA) que necesiten un implante transcatéter de válvula aórtica (TAVI), pacientes con insuficiencia mitral (IM) grave que no puedan ser egresados del hospital de acuerdo con el criterio del grupo de expertos en insuficiencia cardiaca (IC), pacientes con IM grave postinfarto de muy alto riesgo quirúrgico, pacientes con fuga paravalvular en clase funcional III/ IV de la NYHA y/o hemólisis que no puedan ser egresados del hospital de acuerdo con el criterio del grupo de expertos en IC. En pacientes con EA grave asintomáticos, IM grave con adecuada respuesta a tratamiento médico y los que presentan fuga paravalvular en clase I-II se recomienda evaluar caso por caso y estimar la posibilidad de diferir la intervención estructural según sea el caso (Figura 1).

\section{Cardiopatías congénitas}

Se recomienda privilegiar a los pacientes hospitalizados o transferidos de otro hospital (o ambulatorios) que requieran un cateterismo cardiaco urgente debido a compromiso hemodinámico (o compromiso inminente) así como pacientes que se encuentran esperando 


\section{Criterios para la selección de pacientes para procedimientos intervencionistas en salas de cateterismo cardiaco}

\section{Evaluación en salas de urgencias: definir estatus de COVID-19: negativo, no sospechoso, sospechoso o positivo}

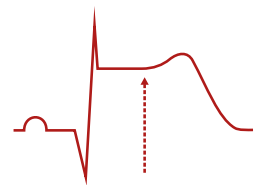

Síndromes coronarios isquémicos agudos IAMCEST O IAMSEST
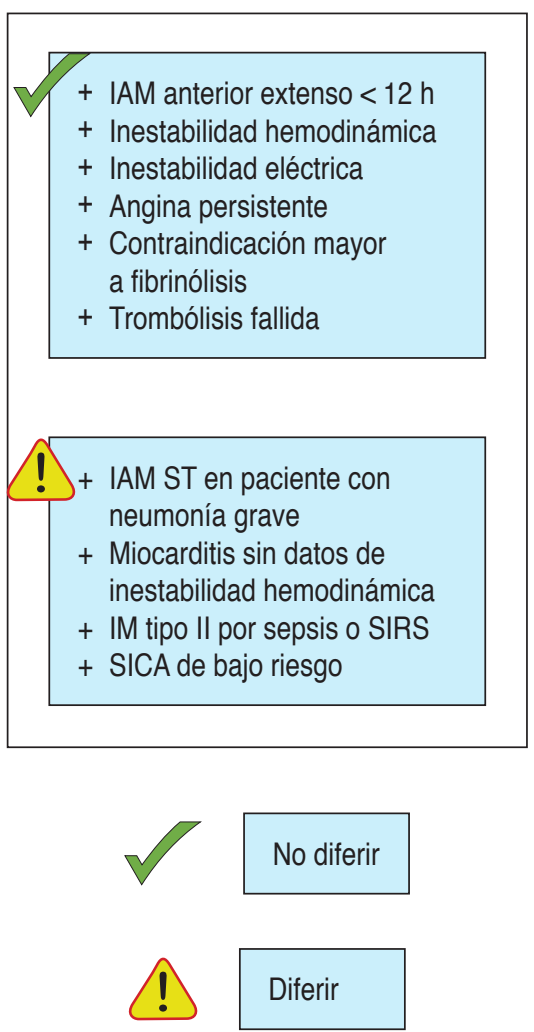
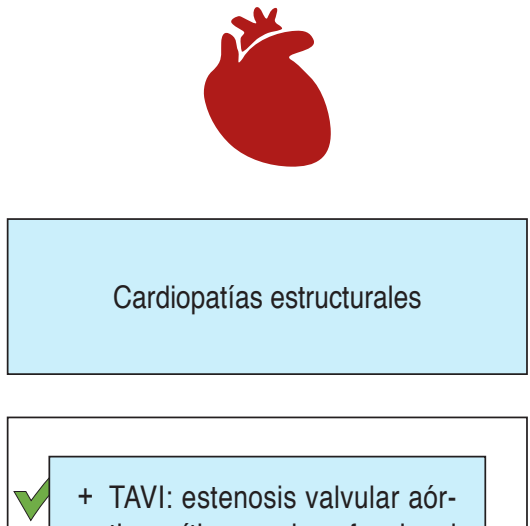

+ TAVI: estenosis valvular aórtica crítica en clase funcional III/IV

+ IM funcional: grave (3/4) que no puede ser egresado del hospital de acuerdo con el criterio del grupo de expertos en insuficiencia cardiaca

+ IM post-IAM: grave (3/4) de muy alto riesgo para cirugía

+ Fuga paravalvular: en clase funcional III/IV y/o hemólisis que no puede ser egresado del hospital de acuerdo al criterio del grupo de expertos en insuficiencia cardiaca

TAVI: pacientes con estenosis valvular aórtica grave asintomáticos

+ IM grave: pacientes que respondan al tratamiento médico

+ Fuga paravalvular: pacientes en clase funcional I-II

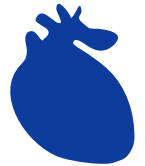

Cardiopatías congénitas

+ Cualquier paciente hospitalizado o paciente trasferido de otro hospital o ambulatorio que requiera un procedimiento de cateterismo cardiaco urgente debido a un compromiso hemodinámico o con compromiso hemodinámico inminente

+ Cualquier paciente esperando un cateterismo cardiaco requerido antes de una cirugía en ese internamiento o requerido un cateterismo para poder egresar a domicilio

+ Cualquier paciente ambulatorio significativamente sintomático 0 aquellos asintomáticos en quienes el diferimiento del procedimiento (> 30 días) pudiera ser deletéreo

Asintomáticos o levemente asintomáticos en quienes se podrían esperar $>1$ mes

Figura 1: Criterios para la selección de pacientes para procedimientos intervencionistas en salas de cateterismo cardiaco.

un cateterismo cardiaco requerido previo a una cirugía o para poder ser egresados del hospital. ${ }^{17}$ También a aquellos pacientes ambulatorios, pero significativamente sintomáticos en quienes un diferimiento mayor de 30 días podría ser deletéreo. ${ }^{17}$ En pacientes asintomáticos o levemente sintomáticos en quienes sería posible esperar más de un mes como externos, se recomienda evaluar el caso para su diferimiento (Figura 1). ${ }^{17}$ 


\section{MEDIDAS DE SEGURIDAD PERSONAL DE LOS PROFESIONALES DE LA SALUD}

\section{La importancia de la protección personal}

Existen múltiples comunicados editoriales y estudios observacionales en los que se ha demostrado que instaurar protocolos de seguridad con el uso apropiado del adecuado equipo de protección personal (EPP) disminuye el riesgo de contagio de los profesionales de la salud y por consecuencia, su riesgo de morbimortalidad. En China se ha reportado hasta 20\% de casos positivos a COVID-19 entre personal de salud. ${ }^{18}$ Recientemente, en Italia, el grupo de Tarantini G. y colaboradores compararon la tasa de contagio de COVID-19 entre el personal de salud de servicios de un mismo hospital, específicamente el servicio de cardiología (incluyendo personal de salas de cateterismo), quienes tenían un protocolo de seguridad frente a la enfermedad de COVID-19 y los compararon con otros servicios del mismo hospital que no tenían un protocolo de seguridad. Tras un seguimiento de 20 días, el personal de salud de los servicios con protocolos de seguridad presentó $1.2 \%$ de casos positivos a COVID-19, mientras que el personal de salud de los servicios sin protocolos de seguridad presentó $12.5 \%$ de casos positivos $(p<0.001) .{ }^{19}$

\section{Consideraciones de seguridad especiales}

Debido al modelo epidemiológico que se usa en nuestro país, será difícil saber qué pacientes son positivos y cuáles negativos, es por eso, y justificado con las altas tasas de contagio, que durante el brote de COVID-19 todo paciente debe ser considerado como positivo hasta que no se demuestre lo contrario. Se deberán diferir todos los procedimientos electivos y sólo se realizarán procedimientos urgentes o casos electivos que no puedan ser diferidos por un tiempo estimado de tres a cuatro meses. ${ }^{15}$

En caso de contar con salas de cateterismo con presión negativa, se deberá priorizar el uso de esa sala. En caso de contar con múltiples salas de cateterismo, centrar la atención de los pacientes en una misma sala. ${ }^{10}$ Todos los traslados de pacientes deberán ser realizados por personal de camillería con adecuado EPP y sería este personal quien coloque al paciente en la mesa del procedimiento y quien retire al paciente de dicha mesa para su traslado al haber finalizado éste. Deben entrar a sala solamente aquellos profesionales de la salud que se consideren indispensables para llevar a cabo el procedimiento; ${ }^{10}$ esto incluye a uno o dos operadores, un anestesiólogo cardiovascular, un personal de enfermería y un técnico radiólogo. Una vez el paciente en la mesa del procedimiento y con la camilla de traslado fuera del área del departamento de cardiología intervencionista, el equipo de profesionales de la salud que participará en el procedimiento iniciará con el vestido (ver más adelante), comenzando con el personal de enfermería para que de esta manera pueda entrar a la sala con el paciente. Los procedimientos tienen que realizarse todo el tiempo a puerta cerrada, y entre tanto nadie debe entrar ni salir de la sala. ${ }^{10}$ Se deberá tener todo el material previsto (incluyendo balones, stents u otros materiales especiales en la medida de lo posible) a ser utilizado antes de comenzar la intervención.

Es importante evaluar a todo paciente que va a ser llevado a sala de cateterismo antes de su traslado. ${ }^{10} \mathrm{~A}$ todos los pacientes se le deberá interrogar en búsqueda de síntomas o signos de COVID-19 así como determinar la presencia o ausencia de fiebre. ${ }^{10}$ De acuerdo con los Centros de Prevención y control de Enfermedades Infecciosas (CDC) de EE.UU., los síntomas más frecuentes reportados por pacientes hospitalizados al inicio de la enfermedad son fiebre, tos, mialgias o fatiga y disnea; sin embargo, se han reportado hasta 17 síntomas relacionados, incluyendo diarrea, anosmia y disgeusia. ${ }^{20}$

\section{Equipo de protección personal}

La importancia de utilizar el adecuado EPP, además de las razones expuestas previamente, radica en el potencial escenario de máximo contagio al cual estaría expuesto todo el personal de salud que interviene en estos procedimientos. ${ }^{6}$ Durante cualquier procedimiento invasivo en una sala de cateterismo cardiaco existe la posibilidad de que un paciente presente deterioro cardiorrespiratorio que amerite manejo avanzado de la vía aérea e intubación orotraqueal urgente, así como maniobras de 
reanimación cardiopulmonar avanzadas, escenarios definidos por la OMS como de máximo riesgo de contagio y que ameritan protección personal máxima. ${ }^{6}$ Es por esto que se requiere EPP de máxima seguridad; ;,10,16 también se recomienda que cualquier otro procedimiento invasivo que pueda ser realizado fuera de sala de cateterismo, se efectúe antes en la sala de urgencias o en la unidad coronaria, según sea el caso (colocación de catéter central, catéter de flotación, de balón de contrapulsación aórtica, marcapaso temporal así como ventilación mecánica invasiva según sea el caso) (Figura 1).

Se recomienda para todo el personal de salud el siguiente EPP (en orden de colocación, ver más adelante): uniforme quirúrgico desechable, mandil de plomo, un par de botas desechables, mascarilla N95 (o de efectividad superior) desechable, mascarilla quirúrgica desechable, gafas de protección de policarbonato que permitan sello hermético idealmente con cubierta antiempañamiento, gorro tipo escafandra impermeable y desechable, dos pares de guantes (tipo quirúrgico estériles para los operadores, para el resto del personal serán no quirúrgicos y no estériles) y batas impermeables desechables (las cuales serán estériles en el caso de los operadores). ${ }^{6,10,14,16}$

\section{Vestido y desvestido}

Es recomendable llegar al hospital con ropa informal, la cual se debe cambiar por un uniforme quirúrgico de tela una vez dentro de las instalaciones del departamento de cardiología intervencionista (se recomienda también traer unos zapatos de plástico o zuecos, los cuales se calzarán también al momento de vestirse con el uniforme quirúrgico de tela). No se deben portar alhajas ni accesorios $y$, antes del equipamiento de protección, verificar que el EPP esté completo y en adecuado estado (Figura 2). ${ }^{10,16}$

Se debe designar un área de vestido para todo el personal de salud. En este lugar se deberá tener preparado todo el EPP listo para el vestido. Ya en el área de vestido, se iniciará con la técnica universal de lavado de manos con agua y jabón para proceder a la colocación del uniforme quirúrgico desechable sobre el uniforme de tela. A continuación, se coloca el mandil de plomo y antes de colocarse el EPP, realizar otro lavado de manos universal. Después se colocarán, en orden ascendente, las botas desechables, luego respirador N95 y la mascarilla quirúrgica encima del respirador N95. Después las gafas protectoras y por último el gorro. Una vez colocado todo esto, el personal de salud que no sea operador, se colocará un primer par de guantes no estériles, seguido de la bata desechable no estéril y por último el segundo par de guantes no estériles. En este momento, el personal ya puede entrar a la sala de cateterismo. Para los operadores, antes de colocarse el primer par de guantes será el momento de realizar el lavado de manos quirúrgico. A continuación, se colocará el primer par de guantes quirúrgicos estériles, seguido de la bata desechable estéril. Una vez vestido de esta manera, el operador podrá entrar a la sala de cateterismo. Una vez dentro y sin haber tocado ninguna superficie, se colocará el segundo par de guantes quirúrgicos estériles (Figura 2).

Una vez finalizado el procedimiento, el desvestido se tiene que hacer en dos etapas, una dentro de sala y otra fuera de ésta. ${ }^{10,16}$ Los siguientes pasos aplican para todo el equipo que entró al procedimiento. Dentro de sala, se inicia con la prerretirada del par de guantes externos, siempre con técnica de pellizco (sin tocar la parte interna de los guantes). A continuación prerretirar la bata, la cual se recoge y enrolla sobre sí misma, de tal manera que se evite que la superficie externa quede en contacto con el medio. Proseguir en un solo movimiento, a retirar bata y guantes externos, los cuales se depositarán en un contenedor de desechos contaminados en el interior de la sala. A continuación, y realizando esto a manera de transferencia entre un área contaminada y un área limpia entre el interior y el exterior de la sala, apoyados con un banco, se retirarán las botas desechables una por una, de tal manera que al retirarse la primera bota el personal pise sin protección el exterior (no contaminado) de la sala, siguiendo ahora los mismos pasos para el otro pie. De esta manera, el personal de salud ahora ya se encuentra fuera de sala. Una vez fuera de sala, se retirará el equipo de manera inversa a la colocación, de arriba hacia abajo. Iniciando por el gorro, tomándolo de la parte más trasera y siempre 
de la parte externa y desechar. A continuación, retirar las gafas de protección, esto cerrando los ojos y tomando la correa de la parte más trasera. Una vez retirados, depositarlos en un contenedor de agua clorada al 10\% (donde deberán permanecer un tiempo aproximado de 20 minutos). Posteriormente, retirar la mascarilla quirúrgica y desecharla, se toma de los extremos más posteriores de las orejas y cerrando los ojos durante el retiro. A continuación, se retirará el mandil de plomo y se colocará en el contenedor. Ahora, se retirará el uniforme quirúrgico desechable y por último el par de guantes interno. Lo último en retirar será el respirador N95, el cual de acuerdo con su disponibilidad podría llegar a ser necesario reutilizar (ver más adelante). Es de vital importancia que entre cada paso del desvestido, incluyendo entre bota y bota, se debe realizar un lavado de guantes con alcohol gel por unos 20-30 segundos. Una vez retirado todo el equipo, realizar un lavado de manos que se extienda hasta los brazos, para así concluir con el protocolo de seguridad (Figura 2). ${ }^{10,16}$

Protocolo de seguridad para un procedimiento invasivo cardiovascular percutáneo vestido y desvestido

ANTES del procedimiento: verificar que se cuenta con todo el material y éste está en condiciones adecuadas

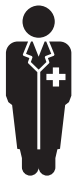

Vestido

Vestido

No-operadores

1. Uniforme quirúrgico desechable*

2. Mandil de plomo*

3. Botas desechables

4. Mascarilla N95

5. Mascarilla quirúrgica

6. Gafas de protección

7. Gorro desechable*

8. Primer par de guantes

9. Bata impermeable

10. Segundo par de guantes

Entrar a sala

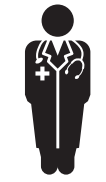

\section{Vestido}

Operadores

1. Uniforme quirúrgico desechable*

2. Mandil de plomo*

3. Botas desechables

4. Mascarilla N95

5. Mascarilla quirúrgica

6. Gafas de protección

7. Gorro desechable***

8. Primer par de guantes

9. Bata impermeable

Entrar a sala

10. Segundo par de guantes
DESPUÉS del procedimiento: la mascarilla N95 es lo último en retirarse

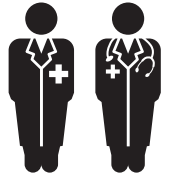

Desvestido
DENTRO de la sala:

1. Pre-retiro de guantes y bata

2. Retiro de guantes y bata*

3. Transfer retirando botas ${ }^{\star *}$

FUERA de la sala:

4. Retiro de gorro**

5. Retiro de gafas-agua clorada*

6. Retiro de mascarilla quirúrgica**

7. Retiro de mandil de plomo**

8. Retiro de quirúrgico desechable**

9. Retiro de guantes internos**

10. Retiro de mascarilla N95*

* Lavado de manos universal con agua y jabón. ** Lavo de manos con alcohol gel. ${ }^{\star \star \star}$ Lavo de manos quirúrgico.

Figura 2: Protocolo de seguridad para un procedimiento invasivo cardiovascular percutáneo. Vestido y desvestido. 


\section{Desinfección del equipo de protección y de la sala de cateterismo}

Una vez finalizado el procedimiento y cuando el personal de salud se encuentre fuera de la sala del procedimiento (excepto personal de enfermería y anestesiología en caso de ser necesario que permanecerá en sala hasta después de la salida del paciente), el personal de camillería trasladará al paciente de la mesa del procedimiento a la camilla y de ahí a su cama de hospitalización. En este momento, el personal restante que se encuentre dentro de sala procederá al protocolo de desvestido. ${ }^{10,16}$

Todo el material utilizado en el procedimiento tiene que ser desechado de manera apropiada. Es importante esperar una hora tras finalizar el procedimiento para que el personal de limpieza pueda iniciar la descontaminación de la sala del procedimiento, para lo cual deberían portar un EPP completo. ${ }^{10}$

En caso de disposición limitada de EPP, en particular de respiradores N95, existen ya diversas técnicas recomendadas por la $\mathrm{OMS}^{6,14}$ y los CDC $^{21}$ y avaladas por los Institutos Nacionales de Salud ${ }^{22}$ de EE.UU. que pueden utilizarse para desinfectar estos respiradores de manera efectiva y sin dañar considerablemente el material o su efectividad como serían la luz ultravioleta, o el tratamiento con calor o peróxido de hidrógeno vaporizado. 6,14,21,22

\section{CONCLUSIONES}

El recorrido que ha tenido la pandemia a lo largo del mundo ha impactado de manera similar en la mayoría de los países y ha obligado a la modificación de los protocolos de atención de los pacientes cardiópatas que ingresan a una sala de cateterismo cardiaco, esto con la finalidad de poder encontrar un equilibro en el dilema de atención de calidad y diminución de riesgos del paciente y de los profesionales de la salud. El mensaje más importante es continuar con la atención de calidad en las salas de cateterismo cardiaco, disminuyendo al máximo los riesgos de contagio de los pacientes y del personal de salud, riesgos que se ha demostrado pueden disminuirse hasta 10 veces con el uso de protocolos de seguridad y del apropiado equipo de protección personal.

\section{REFERENCIAS}

1. Zhu N, Zhang DY, Wang WL, Li X, Yang B, Song J et al. A novel coronavirus from patients with pneumonia in China, 2019. N Engl J Med. 2020; 382: 727-733.

2. Coronavirus disease 2019 (COVID-19) Situation Report-51 [Internet]. World Health Organization. 11 de marzo de 2020. Disponible en: https://www.who. int/emergencies/diseases/novel-coronavirus-2019/ situation-reports

3. Conferencia de prensa por COVID-19 del 28 de febrero de 2020 [Internet]. México: Secretaría de Salud, Gobierno de México; 28 de febrero de 2020. Disponible en: https://coronavirus.gob.mx/noticias

4. Conferencia de prensa por COVID-19 del 16 de abril de 2020 [Internet]. México: Secretaría de Salud, Gobierno de México; 16 de abril de 2020. Disponible en: https://coronavirus.gob.mx/noticias

5. Infection prevention and control during health care when novel coronavirus ( $\mathrm{nCoV}$ ) infection is suspected. Interim guidance 06 April 2020 [Internet]. World Health Organization; 6 de abril de 2020. Disponible en: https://www.who.int/publications-detail/ infection-prevention-and-control-during-health-carewhen-novel-coronavirus-(ncov)-infection-is-suspected-20200125

6. Rational use of personal protective equipment for coronavirus disease (COVID-19) and considerations during severe shortages. Interim guidance 06 April 2020 [Internet]. World Health Organization; 6 de abril de 2020. Disponible en: https://www.who.int/ publications-detail/rational-use-of-personal-protective-equipment-for-coronavirus-disease-(covid-19)and-considerations-during-severe-shortages

7. Welt FGP, Shah PB, Aronow HD, Bortnick AE, Henry TD, Sherwood MW et al.; American College of Cardiology's (ACC) Interventional Council and the Society of Cardiovascular Angiography and Intervention (SCAI). Catheterization laboratory considerations during the coronavirus (COVID-19) pandemic: from ACC's Interventional Council and SCAI. J Am Coll Cardiol. 2020 Mar 16. doi: 10.1016/j.jacc.2020.03.021. [Epub ahead of print]

8. Han $Y$, Zeng $H$, Jiang $H$, Yang $Y$, Yuan Z, Cheng X et al. CSC Expert consensus on principles of clinical management of patients with severe emergent cardiovascular diseases during the COVID-19 epidemic. Circulation. 2020 Mar 27. doi: 10.1161/CIRCULATIONAHA.120.047011. [Epub ahead of print]

9. Shah PB, Welt FGP, Mahmud E, Phillips A, Kleiman NS, Young MN et al.; from the American College of Cardiology (ACC) and the Society for Cardiovascular Angiography and Interventions (SCAI). Triage considerations for patients referred for structural heart disease intervention during the coronavirus disease 2019 (COVID-19) pandemic: An ACC/SCAI consensus statement. Catheter Cardiovasc Interv. 2020 Apr 6. doi: 10.1002/ccd.28910. [Epub ahead of print]

10. Gestión de salas de procedimientos invasivos cardiológicos durante el brote de coronavirus COVID-19. Documento de consenso de la Asociación de Cardiología Intervencionista y la Asociación del Ritmo Cardiaco de la Sociedad Española de Cardiología 
[Internet]. Sociedad Española de Cardiología; 16 de marzo de 2020. Disponible en: https://secardiologia. es/blog/rec-interventional-cardiology/11449-covid19-documento-de-consenso-gestion-de-salas-deprocedimientos-invasivos-cardiologicos-durante-elbrote-de-coronavirus-covid-19

11. Coronavirus disease (COVID-19) outbreak: rights, roles and responsibilities of health workers, including key considerations for occupational safety and health. Interim guidance 19 March 2020 [Internet]. World Health Organization; 19 de marzo de 2020. Disponible en: https://www. who.int/publications-detail/ coronavirus-disease-(covid-19)-outbreak-rights-rolesand-responsibilities-of-health-workers-including-keyconsiderations-for-occupational-safety-and-health

12. Considerations in the investigation of cases and clusters of COVID-19. Interim guidance 13 March 2020 [Internet]. World Health Organization; 13 de marzo de 2020. Disponible en: https://www.who.int/whodocuments-detail/considerations-in-the-investigationof-cases-and-clusters-of-covid-19

13. Considerations for quarantine of individuals in the context of containment for coronavirus disease (COVID-19). Interim guidance 19 March 2020 [Internet]. World Health Organization; 19 de marzo de 2020. Disponible en: https://www.who.int/publicationsdetail/considerations-for-quarantine-of-individuals-inthe-context-of-containment-for-coronavirus-disease(covid-19)

14. Advice on the use of masks in the context of COVID-19. Interim guidance 06 April 2020 [Internet]. World Health Organization; 6 de abril de 2020. Disponible en: https://www.who.int/publications-detail/ advice-on-the-use- of-masks-in-the-community-during-home-care-and-in-healthcare-settings-in-the-context-of-the-novel-coronavirus-(2019-ncov)-outbreak

15. Critical preparedness, readiness and response actions for COVID-19. Interim guidance 19 March 2020 [Internet]. World Health Organization; 19 de marzo de 2020. Disponible en: https://www.who.int/ publications-detail/critical-preparedness-readinessand-response-actions-for-covid-19

16. Tarantini G, Fraccaro C, Chieffo A, Marchese A, Tarantino FF, Rigattieri $\mathrm{S}$ et al.; GISE. Italian Society of Interventional Cardiology (GISE) position paper for Cath lab-specific preparedness recommendations for healthcare providers in case of suspected, probable or confirmed cases of COVID-19. Catheter Cardiovasc Interv. 2020 Mar 29. doi: 10.1002/ccd.28888. [Epub ahead of print]

17. Morray BH, Gordon BM, Crystal MA, Goldstein BH, Qureshi AM, Torres AJ et al. Resource allocation and decision making for pediatric and congenital cardiac catheterization during the novel coronavirus SARS-CoV-2 (COVID-19) pandemic: A U.S. multi- institutional perspective. J Invasive Cardiol. 2020; 32 (5): E103-E109.

18. Remuzzi A, Remuzzi G. COVID-19 and Italy: what next? Lancet. 2020; 395 (10231): 1225-1228.

19. Tarantini $G$ et al. The role of covid-19 protocol within a cardiology department. Presented at C3 and COVID19 Webinar Series - European Experience and Cardiology. C3 Interventional Academy. 2020 Apr 17. [Por ser publicado]

20. Evaluating and Testing Persons for Coronavirus Disease 2019 (COVID-19) [Internet]. Centers for Disease Control and Prevention; 14 de marzo de 2020. Disponible en: https:/www.cdc.gov/coronavirus/2019-nCoV/hcp/ clinical-criteria.html

21. Decontamination and Reuse of Filtering Facepiece Respirators [Internet]. Centers for Disease Control and Prevention; 9 de abril de 2020. Disponible en: https://www.cdc.gov/coronavirus/2019-ncov/hcp/ppestrategy/decontamination-reuse-respirators.html

22. Fischer R, Morris D, Doremalen $\mathrm{N}$ et al. Assessment of N95 respirator decontamination and re-use for SARS-CoV-2 [Internet]. medR $\chi i v ; 24$ de abril de 2020. Disponible en: https://www.medrxiv.org/content/10.1 101/2020.04.11.20062018v2

\section{Correspondencia: \\ Guering Eid-Lidt}

Departamento de Hemodinamia del Instituto Nacional de Cardiología «lgnacio Chávez».

Juan Badiano Núm. 1,

Col. Belisario Domínguez,

Sección XVI, Tlalpan, 14080,

Ciudad de México, México.

E-mail: guering@yahoo.com

\section{RESPONSABILIDADES ÉTICAS}

Protección de personas y animales. Los autores declaran que para esta investigación no se han realizado experimentos en seres humanos ni en animales.

Confidencialidad de los datos. Los autores declaran que han seguido los protocolos de su centro de trabajo sobre la publicación de datos de pacientes.

Derecho a la privacidad y consentimiento informado. Los autores han obtenido el consentimiento informado de los pacientes $y / o$ sujetos referidos en el artículo. Este documento obra en poder del autor de correspondencia. 